\title{
The inaugural Nano Research Young Innovators (NR45) Award in nanobiotechnology
}

\author{
Zhen $\mathrm{Gu}^{1,2}(\varangle)$ and Hongjie Dai ${ }^{3}(\square)$ \\ ${ }^{1}$ Department of Bioengineering, University of California, Los Angeles, CA 90095, USA \\ ${ }^{2}$ California NanoSystems Institute (CNSI), Jonsson Comprehensive Cancer Center, Center for Minimally Invasive Therapeutics, University \\ of California, Los Angeles, CA 90095, USA \\ ${ }^{3}$ Department of Chemistry, Stanford University, Stanford, CA 94305, USA
}

(C) Tsinghua University Press and Springer-Verlag GmbH Germany, part of Springer Nature 2018

It is our great pleasure to announce awardees of the inaugural 2018 Nano Research Young Innovators (NR45) in nanobiotechnology. Congratulations to all of the 45 outstanding young investigators under 45 ! They were selected through a competitive process by an award committee from our editorial board. Nano Research is launching the NR45 Award program to young researchers in various fields of nanoscience and nanotechnology, in recognition to their distinguished accomplishments and/or potential to make substantial contributions to their fields. The aim of Nano Research NR45 is to recognize the outstanding contributions of young scientists and together with the Nano Research Symposium integrated in the annual US-SINO Nano Forum provide a platform for communication, discussions and collaborations between scientists internationally. For this inaugural year, we highlight 45 innovators in the field of nanobiotechnology, covering research focuses including imaging, drug delivery, and tissue engineering for diagnosis and treatment of different diseases. Their contributions to this special issue contain 28 review articles and 17 research articles.

The special issue begins with reviews about advances in the field of nucleic acid delivery. Ding and coworkers summarize recent achievements in the development of multifunctional nucleic acid nanostructures, including RNA interference, clustered regularly interspaced short palindromic repeat associated proteins 9 system
(CRISPR/Cas9) and CpG for gene delivery [1]. Shi and coworkers focus on messenger RNA (mRNA) delivery strategies for improvement of the stability of mRNA, minimize immune responses, and enhance translational efficacy [2]. They also highlight the recent progress in nanoparticle (NP)-based mRNA delivery. Sun and coworkers provide an overview of recent mRNA vaccine delivery systems, emphasizing the necessity of formulating mRNA vaccines with delivery systems [3]. Lee and coworkers highlight the role of mRNA vaccines as prophylactic vaccines for the infection prevention and as therapeutic vaccines for cancer immunotherapy [4]. Siegwart and coworkers outline the physical and biological challenges of delivering RNA therapeutics, and summarize the criteria to design clinically-relevant nanomaterials required to overcome those challenges [5]. Miyata and coworkers review the use of silica NPs for nucleic acid delivery [6].

Several reviews are focusing on the polymers or polymeric platforms for biomedical applications. For examples, Du and coworkers survey recent advances in the polymerization methodologies for improved therapeutic performance of the resultant nanomedicines [7]. Wang and coworkers focus on the temperature-responsive polymers and their biomedical applications [8]. Cao and coworkers review different strategies, including crosslinking and non-crosslinking

Address correspondence to Zhen Gu, guzhen@ucla.edu; Hongjie Dai, hdai1@stanford.edu 
approaches, to stabilize polymeric micelles for drug delivery [9]. Zhang and coworkers focus on the effects of poly(ethylene glycol) (PEG) architecture on the physiochemical and biological properties of the PEGylated oligonucleotides [10].

Day and coworkers survey the biomimetic NPs for targeted cancer theranostics [11], while Wang and coworkers review the various mechanisms for phototriggered targeting of drug nanocarriers [12]. Besides cancer, Zhang and coworkers summarize various delivery systems for the theranostics in neurodegenerative diseases [13]. Kim and coworkers discuss the current representative studies of high-density lipoprotein (HDL) mimetic nanotherapeutics for cardiovascular and neurodegenerative diseases [14].

Four of the Young Innovator articles discuss the formulations for bioimaging or diagnostics. Landry and coworkers summarize the current methods used for brain microscopy and describe the diverse classes of nanomaterials as contrast agents and functional probes for microscopic optical imaging of the brain [15]. Wei and coworkers highlight the recent advances and applications of plasmonic technologies and discuss the implementation and penetration of various plasmonic technologies in conventional molecular diagnostic assays [16]. Pu and coworkers summarize the design principles and advantages of the reactive oxygen and nitrogen species (RONS)-responsive activatable nanoprobes, as well as their applications in different diseases [17]. Yuan and coworkers stratify the recent progress in background-free latent fingerprint imaging by making use of the difference in properties between contrast agents and background compounds [18].

Other delivery methodologies, such as cell-based delivery systems, metal-based nano-formulations, and inorganic NPs, are also discussed. Mo and coworkers present recent advances in cell-based systems for drug delivery, tissue engineering, bio-imaging, and disease treatment [19]. Xing and coworkers focus on the specificity and environment-responsiveness of metallodrug nanoformulations as therapeutics, and summarize the recent strategies to develop metal complex-functionalized intelligent nanoplatforms [20]. Anselmo and coworkers describe the role of inorganic NPs, especially those that are encountered in everyday life, in shaping and influencing commensal microbiota [21].

Nano/bio-materials for cell and tissue engineering are also featured in this special issue. Imoukhuede and coworkers summarize the methodologies for surface functionalization on the glass bottoms and their applications to wide-ranges of cell investigations, including cell patterning and cell isolation [22]. Ekenseair and coworkers highlight the theory and fabrication of magneto-responsive scaffolds for applications in tissue regeneration and artificial muscles, as well as drug delivery [23]. Timko and coworkers focus specifically on one-dimensional (1D) nanomaterials and discuss the nature of the nanomaterial-cell interface, and how that interface can be engineered to modulate cellular function [24].

Functional materials for other applications are also covered. For example, Tang and coworkers provide an overview of the recent advances in applying stimuliresponsive biomaterials in enhancing non-cellular cancer vaccines while with an emphasis on the chemistry and material design with varied responsiveness [25]. Cai and coworkers review the types of reactive oxygen and nitrogen species, how they are implicated in several diseases, and the types of NPs with inherent antioxidant capability, their mechanisms of action, and their biological applications [26]. Guo and coworkers survey the development of functional neural interfaces and their applications on the nervous systems [27]. Lovell and coworkers summarize several clinical-stage approaches for taxane formulations, and recent efforts to develop novel cabazitaxel delivery systems for cancer therapy [28].

These review articles are followed by 17 research articles covering various types of biomaterials for a wide range of biomedical applications. $\mathrm{Wu}$ and coworkers report binary transition metal oxide-based Janus NP via a facial fabrication process for cancer theranostics [29]. He and coworkers describe a new traditional Chinese medicine molecule-assisted chemical synthetic strategy to prepare ultrasmall fluorescent silica NPs for cancer treatment [30]. Liu and coworkers present a unique design comprising an albumin/catalase co-modified multifunctional $\mathrm{Au}$ nanocluster-based theranostic nanoplatform for simultaneous fluorescence imaging and the second near-infrared (NIR-II) light activated photodynamic therapy (PDT) [31]. Wang 
and coworkers report a nanomedicine capable of hierarchical targeting with improved tumor accumulation and enhanced tumor retention, as well as nuclear translocation of encapsulated platinum prodrugs [32]. Jewell and coworkers describe a polyplex system formed by $\mathrm{CpG}$ and polycation (poly( $\beta$-amino ester)) for cancer immunotherapy and study the impact of the polyplex interaction strength on immune responses [33].

$\mathrm{Xie}$ and coworkers engineer a $\mathrm{LiF}_{\mathrm{SiO}}$ nanocapsule for the sustained delivery of lithium to treat osteoarthritis [34]. Nance and coworkers show that curcumin-loaded polymeric NPs exhibit a considerable neuroprotective effect in a hypoxic-ischemic brain injury rat model [35]. Gao and coworkers study on the shape effect of E3-reconstituted high-density lipoprotein (rHDL) on the brain delivery, and demonstrate that spherical rHDL exhibits better capabilities in reducing $A \beta$ deposition and rescuing memory deficits in a mouse model of Alzheimer's disease [36]. Chung and coworkers describe a kidney-targeting peptide-modified multimodal micelle as a potential drug nanocarrier for kidney diseases [37].

Scott and coworkers demonstrate the scalable production of poly(ethyleneglycol)-block-poly(propylene sulfide) polymersomes via flash nanoprecipitation and further show the safe administration of these nanocarriers to mice and non-human primates [38]. Cheng and coworkers show that red blood cell membrane coating on the poly(lactic-co-glycolic acid) (PLAG) NPs could attenuate the inflammation response induced by the bare NPs [39].

Dong and coworkers reported an amino-ester-based nanomaterial for the codelivery of mRNA and superparamagnetic iron oxide nanoparticles (SPIONs) with enhanced mRNA delivery efficiency and MRI contrast signals [40]. Chen and coworkers present microribbons formed by self-assembly of Human Galectin-1 (Gal-1) with small molecule ligands, and show that protein microribbons interestingly inhibit Gal-1 induced T-cell agglutination and apoptosis [41]. Cao and coworkers design a photo-switchable hydrogel platform with spatiotemporally tunable mechanical properties, which can be used to assay and define cellular behaviors through lights [42]. Wang and coworkers report a subcellular-patterned biointerface from self-assembled opal films to manipulate the surface wetting of multicellular spheroids by simply altering the sizes of colloidal particles, suggesting that the surface topography is another factor that affects the wetting of multicellular spheroids and provides new approaches for the control of tissue wetting velocity [43]. Fang and coworkers design a new type of neural electrodes made of hot-pressed nanopaper substrates for neutral activity recording, which show enhanced biocompatibility, high flexibility, and good shape stability [44]. Xu and coworkers report a general and facile method to fabricate glutathione peroxidase (GPx) mimic metal-organic frameworks (MOFs) that exhibit excellent catalytic activity and thermal and structural stability [45].

Overall, the collection of this issue highlights the diverse topics on the nanobiotechnology for a broad spectrum of biomedical applications. We sincerely thank all the awardees for their excellent contributions to this inaugural NR45 Special Issue. We hope that you will enjoy this special issue, and encourage you and your colleagues nominate young innovators in your field for following years' NR45 award competition.

\section{References}

[1] Liu, J.; Wang, Z.; Zhao, S.; Ding, B. Multifunctional nucleic acid nanostructures for gene therapies. Nano Res. 2018, 11, 5017-5027.

[2] Xiong, Q.; Lee, G. Y.; Ding, J.; Li, W.; Shi, J. Biomedical applications of mRNA nanomedicine. Nano Res. 2018, 11, 5281-5309.

[3] Tan, L.; Sun, X. Recent advances in mRNA vaccine delivery. Nano Res. 2018, 11, 5338-5354.

[4] Lee, K.; Kim, M.; Seo, Y.; Lee, H. Development of mRNA vaccines and their prophylactic and therapeutic applications. Nano Res. 2018, 11, 5173-5192.

[5] Miller, J. B.; Siegwart, D. J. Design of synthetic materials for intracellular delivery of RNAs: From siRNA-mediated gene silencing to CRISPR/Cas gene editing. Nano Res. 2018, 11, 5310-5337.

[6] Kamegawa, R.; Naito, M.; Miyata, K. Functionalization of silica nanoparticles for nucleic acid delivery. Nano Res. 2018, 11, 5219-5239.

[7] Jia, X.; Wan, L.; Du, J. In situ polymerization on biomacromolecules for nanomedicines. Nano Res. 2018, 11, 5028-5048. 
[8] Qiao, S.; Wang, H. Temperature-responsive polymers: Synthesis, properties, and biomedical applications. Nano Res. 2018, 11, 5400-5423.

[9] Lu, Y.; Zhang, E.; Yang, J.; Cao, Z. Strategies to improve micelle stability for drug delivery. Nano Res. 2018, 11, 4985-4998.

[10] Lu, X.; Zhang, K. PEGylation of therapeutic oligonucletides: From linear to highly branched PEG architectures. Nano Res. 2018, 11, 5519-5534.

[11] Valcourt, D. M.; Harris, J.; Riley, R. S.; Dang, M.; Wang, J.; Day, E. S. Advances in targeted nanotherapeutics: From bioconjugation to biomimicry. Nano Res. 2018, 11, 49995016.

[12] Li, Y.; Zhang, Y.; Wang, W. Phototriggered targeting of nanocarriers for drug delivery. Nano Res. 2018, 11, 5424 5438.

[13] Li, Y.; Liu, R.; Ji, W.; Li, Y.; Liu, L.; Zhang, X. Delivery systems for theranostics in neurodegenerative diseases. Nano Res. 2018, 11, 5535-5555.

[14] Ahn, S. I.; Park, H.-J.;Yom, J.; Kim, T.; Kim, Y. T. Highdensity lipoprotein mimetic nanotherapeutics for cardiovascular and neurodegenerative diseases. Nano Res. 2018, 11, 5130-5143.

[15] Del Bonis-O'Donnell, J. T.; Chio, L.; Dorlhiac, G. F.; McFarlane, I. R.; Landry, M. P. Advances in nanomaterials for brain microscopy. Nano Res. 2018, 11, 5144-5172.

[16] Yu, T.; Wei, Q. Plasmonic molecular assays: Recent advances and applications for mobile health. Nano Res. 2018, 11, 5439-5473.

[17] Zhen, X.; Pu, K. Development of optical nanoprobes for molecular imaging of reactive oxygen and nitrogen species. Nano Res. 2018, 11, 5258-5280.

[18] Wang, Y.; Wang, J.; Ma, Q.; Li, Z.; Yuan, Q. Recent progress in background-free latent fingerprint imaging. Nano Res. 2018, 11, 5499-5518.

[19] Li, T.; Dong, H.; Zhang, C.; Mo, R. Cell-based drug delivery systems for biomedical applications. Nano Res. 2018, 11, 5240-5257.

[20] Hu, M.; Ai, X.; Wang, Z.; Zhang, Z.; Cheong, H.; Zhang, W.; Lin, J.; Li, J.; Yang, H.; Xing, B. Nanoformulation of metal complexes: Intelligent stimuli-responsive platforms for precision therapeutics. Nano Res. 2018, 11, 5474-5498.

[21] Qiu, K.; Durham, P. G.; Anselmo, A. C. Inorganic nanoparticles and the microbiome. Nano Res. 2018, 11, 49364954.

[22] Ansari, A.; Imoukhuede, P. I. Plenty more room on the glass bottom: Surface functionalization and nanobiotechnology for cell isolation. Nano Res. 2018, 11, 5107-5129.
[23] Adedoyin, A. A.; Ekenseair, A. K. Biomedical applications of magneto-responsive scaffolds. Nano Res. 2018, 11, 50495064.

[24] Liu, H.; Haider, B.; Fried, R. H.; Ju, J.; Bolonduro, O.; Raghuram, V.; Timko, P. B. Nanobiotechnology: 1D nanomaterial building blocks for cellular interfaces and hybrid tissues. Nano Res. 2018, 11, 5372-5399.

[25] Zhao, Y.; Guo, Y.; Tang, L. Engineering cancer vaccines using stimuli-responsive biomaterials. Nano Res. 2018, 11, 5355-5371.

[26] Ferreira, C. A.; Ni, D.; Rosenkrans, Z. T.; Cai, W. Scavenging of reactive oxygen and nitrogen species with nanomaterials. Nano Res. 2018, 11, 4955-4984.

[27] Wang, Y.; Zhu, H.; Yang, H.; Argall, A. D.; Luan, L.; Xie, C.; Guo, L. Nano functional neural interfaces. Nano Res. 2018, 11, 5065-5106.

[28] Sun, B.; Straubinger, R. M.; Lovell, J. F. Current taxane formulations and emerging cabazitaxel delivery systems. Nano Res. 2018, 11, 5193-5218.

[29] Iqbal, M. Z.; Ren, W.; Saeed, M.; Chen, T.; Ma, X.; Yu, X.; Zhang, J.; Zhang, L.; Li, A.; Wu, A. A facile fabrication route for binary transition metal oxide-based Janus nanoparticles for cancer theranostic applications. Nano Res. 2018, 11, 5735-5750.

[30] Ji, X.; Guo, D.; Song, B.; Wu, S.; Chu, B.; Su, Y.; He, Y. Traditional Chinese medicine molecule-assisted chemicalsynthesis of fluorescent anti-cancer silicon nanoparticles. Nano Res. 2018, 11, 5629-5641.

[31] Chen, Q.; Chen, J.; Yang, Z.; Zhang, L.; Dong, Z.; Liu, Z. NIR-II light activated photodynamic therapy with proteincapped gold nanoclusters. Nano Res. 2018, 11, 5657-5669.

[32] Jiang, W.; Wang, J.; Yang, J.; He, Z.; Hou, Z.; Luo, Y.; Wang, L.; Liu, J.; Zhang, H.; Zhao, Y. et al. Aciditytriggered TAT-presenting nanocarriers augment tumor retention and nuclear translocation of drugs. Nano Res. 2018, 11, 5716-5734.

[33] Tsai, J. S.; Andorko, I. J.; Zeng, X.; Gammon, M. J.; Jewell, M. C. Polyplex interaction strength as a driver of potency during cancer immunotherapy. Nano Res. 2018, 11, 56425656.

[34] Todd, T.; Lu, Z.; Zhao, J.; Cline, B.; Zhang, W.; Chen, H.; Kumar, A.; Jiang, W.; West, F.; Franklin, S. et al. LiF@ $\mathrm{SiO}_{2}$ nanocapsules for controlled lithium release and osteoarthritis treatment. Nano Res. 2018, 11, 5751-5760.

[35] Joseph, A.; Wood, T.; Chen, C.-C.; Corry, K.; Snyder, J. M.; Juul, S. E.; Parikh, P.; Nance, E. Curcumin-loaded polymeric nanoparticles for neuroprotection in neonatal rats with hypoxic-ischemic encephalopathy. Nano Res. 2018, 11, 56705688 
[36] Song, H.; Ma, X.; Xu, J.; Song, Q.; Hu, M.; Gu, X.; Zhang, Q.; Hou, L.; Chen, L.; Huang, Y. et al. The shape effect of reconstituted high-density lipoprotein nanocarriers on brain delivery and A $\beta$ clearance. Nano Res. 2018, 11, 5615-5628.

[37] Wang, J.; Poon, C.; Chin, D.; Milkowski, S.; Lu, V.; Hallows, K. R.; Chung, E. J. Design and in vivo characterization of kidney-targeting multimodal micelles for renal drug delivery. Nano Res. 2018, 11, 5584-5595.

[38] Allen, S. D.; Liu, Y.-G.; Bobbala, S.; Cai, L.; Hecker, P. I.; Temel, R.; Scott, E. A. Polymersomes scalably fabricated via flash nanoprecipitation are non-toxic in non-human primates and associate with leukocytes in the spleen and kidney following intravenous administration. Nano Res. 2018, 11, 5689-5703.

[39] Fan, Z.; Li, P. Y.; Deng, J.; Bady, S. C.; Cheng, H. Cell membrane coating for reducing nanoparticle-induced inflammatory responses to scaffold constructs. Nano Res. 2018, 11, 5573-5583.

[40] Luo, X.; Zhao, W.; Li, B.; Zhang, X.; Zhang, C.; Bratasz, A.; Deng, B.; McComb, D. W.; Dong, Y. Co-delivery of mRNA and SPIONs through amino-ester nanomaterials. Nano Res. 2018, 11, 5596-5603.
[41] Qi, W.; Zhang, Y.; Kochovski, Z.; Wang, J.; Lu, Y.; Chen, G.; Jiang, M. Self-assembly of Human Galectin-1 via dual supramolecular interactions and its inhibition of T-cell agglutination and apoptosis. Nano Res. 2018, 11, 55665572.

[42] Wu, X.; Huang, W.; Wu, W.-H.; Xue, B.; Xiang, D.; Li, Y.; Qin, M.; Sun, F.; Wang, W.; Zhang, W.-B. et al. Reversible hydrogels with tunable mechanical properties for optically controlling cell migration. Nano Res. 2018, 11, 5556-5565.

[43] Wang, L.; Cai, P.; Luo, J.; Zhang, F.; Liu, J.; Chen, Y.; Zhu, Z.; Song, Y.; Yang, B.; Liu, X. et al. Engineering subcellularpatterned biointerfaces to regulate the surface wetting of multicellular spheroids. Nano Res. 2018, 11, 5704-5715.

[44] Guo, Y.; Fang, Z.; Du, M.; Yang, L.; Shao, L.; Zhang, X.; Li, L.; Shi, J.; Tao, J.; Wang, J. et al. Flexible and biocompatible nanopaper-based electrode arrays for neural activity recording. Nano Res. 2018, 11, 5604-5614.

[45] Zhou, W.; Li, H.; Xia, B.; Ji, W.; Ji, S.; Zhang, W.; Huang, W.; Huo, F.; Xu, H. Selenium-functionalized metal-organic frameworks as enzyme mimics. Nano Res. 2018, 11, 57615768 . 